Pacific Journal of Mathematics

PROXIMITY APPROACH TO SEMI-METRIC AND

ANI GAGRAT AND SOMASHEKHAR AMRITH NAIMPALIY 


\section{PROXIMITY APPROACH TO SEMI-METRIC AND DEVELOPABLE SPACES}

\section{Gagrat and S. A. NAImpally}

In this paper we study semi-metric and developable spaces via generalized proximities and uniformities. We find sufficient conditions for a compatible semi-metric $d$ on a space $X$ to induce a Lodato proximity and also study the effects on a space $X$ when $d$ satisfies various weaker forms of continuity. We present two new characterizations of developable spaces, one of which reads: $A T_{1}$-space is developable if and only if it has a compatible upper semi-continuous semimetric.

We give improved versions of two known metrization theorems. Finally, we generalize the concepts: $T_{1}$-map, uniform map, completely uniform map, pseudo-open map, etc., to apply to proximity spaces and improve some of the known results; for example, an open uniform image of a developable space is developable.

Our motivation for this study was a result mentioned by Arkhangel'skii [4]: A semimetric space $(X, d)$ is metrizable if the induced nearness relation $\delta(d)$ is an $E F$-proximity (where $A \delta(d) B$ iff $d(A, B)=\operatorname{Inf}\{d(a, b): a \in A, b \in B\}=0)$. One naturally wonders as to what properties are satisfied by $X$ if $\delta(d)$ satisfies weaker proximity axioms such as those of an $S$-, a $L O$ - or an $R$-proximity. We soon found that $\delta(d)$ is always an $S$-proximity and that, if $d$ is the natural semi-metric associated with a developable space, then $\delta(d)$ is a $L O$-proximity. Further impetus was provided by a result due to Pareek [15]: A $T_{1}$-space is semi-metrizable iff there exists a countable family $\left\{W_{n}: n \in N\right\}$ of symmetric subsets of $X \times X$ satisfying (a) $\bigcap_{n=1}^{\infty} W_{n}=$ $\Delta$ and (b) for each $x \in X,\left\{W_{n}[x]: n \in N\right\}$ forms a neighbourhood base at $x$. This result is analogous to the well-known Alexandroff-Urysohn theorem: A $T_{1}$-space is metrizable iff it has a compatible uniformity with a countable base. This provided us with a motivation to find one of our new characterizations of developable spaces.

The basic definitions and results are given in this section. In the next section, we study semi-metrizable spaces having compatible semi-metric which satisfy various weaker forms of continuity, e.g., those which are lower semi-continuous or are separately continuous. We also find sufficient conditions for $\delta(d)$ to be a $L O$ proximity.

In the third section, we obtain two new characterizations of developable spaces and also find a sufficient condition for a space to 
be a totally bounded developable space. In $\S 4$, we prove two new metrization theorems. Finally, in the fifth section, we study the images of some specific proximity spaces under some special mappings.

We now give some basic definitions and results which are needed in this paper. Let $X$ be a nonempty set and let $\delta$ be a binary relation on the power set of $X$. Consider the following axioms:

1.1. ( i ) $A \delta B$ implies $B \delta A$,

(ii ) $A \delta(B \cup C)$ iff $A \delta B$ or $A \delta C$,

(iii ) $A \delta B$ implies $A \neq \phi, B \neq \phi$,

(iv) $A \cap B \neq \phi$ implies $A \delta B$,

( v ) $a \delta B$ and $b \delta C$ for every $b \varepsilon B$ implies $a \delta C$,

(vi ) $A \delta B$ and $b \delta C$ for every $b \varepsilon B$ implies $A \delta C$,

(vii) $a \Phi B$ implies the existence of an $E \subset X$ such that $a \phi E$ and $(X-E) \Phi B$,

(viii) $A \Phi B$ implies the existence of an $E \subset X$ such that $A \Phi E$ an $(X-E) \oiint B$.

\section{DEFINITION 1.2. The relation $\delta$ is called}

(a) an S-proximity if it satisfies 1.1 (i)-(iv) and (v) (see [13]),

(b) a LO-proximity if it satisfies 1.1 (i)-(iv) and (vi) (see [13]),

(c) an $R$-proximity if it satisfies 1.1 (i)-(iv) and (vii) (see [11]),

(d) an EF-proximity if it satisfies 1.1 (i)-(iv) and (viii) (see [13]).

By a "proximity $\delta$ ", we mean any one of the four proximities defined above and in this case we call the pair $(X, \delta)$ a proximity space. A binary relation $\delta$ that satisfies 1.1 (i)-(iv) induces an operator $A \rightarrow \bar{A}=\{x \in X: x \delta A\}$ on the power set of $X$, and this will be a Kuratowski closure operator if and only if $\delta$ is an $S$-proximity. Since (viii) implies (vii), (viii) implies (vi), and (vii) or (vi) implies (v), then every proximity is an $S$-proximity. The topology induced by the closure operator of a proximity $\delta$ will be written $\tau(\delta)$.

We assume that all proximities $\delta$ considered in this paper are separated, i.e., xoy implies $x=y$. Obviously in this case $\tau(\delta)$ is $T_{1}$.

If $(X, \tau)$ is a topological space and $\delta$ is a proximity on $X$ such that $\tau=\tau(\delta)$, then we say that $\tau$ and $\delta$ are compatible. It is known that: (i) every $T_{1}$-space has a compatible $L O$ - (and ipso facto $S$-) proximity [13]; (ii) A topological space is $T_{3}$ iff it has a compatible $R$-proximity [11]; (iii) A topological space is Tychonoff iff it has a compatible $E F$-proximity.

Definition 1.3. A semi-metric space $(X, d)$ is a $T_{1}$-space $X$ together with a real-valued function $d$ on $X \times X$ such that

(a) $d(x, y)=d(y, x) \geqq 0$, 
(b) $d(x, y)=0$ iff $x=y$,

(c) $x \in M^{-}$iff $d(x, M)=0$, where $d(A, B)$

$$
\begin{aligned}
& =\operatorname{Inf}\{d(a, b): a \in A, b \in B\}, \text { if } A \neq \phi \neq B \\
& =\infty \text { if } A=\phi \text { or } B=\phi .
\end{aligned}
$$

If $(X, d)$ is a semi-metric space, we define $\delta=\delta(d)$ by

$$
A \delta B \text { iff } d(A, B)=0 \text {. }
$$

It is easily verified that $\delta(d)$ is an $S$-proximity on $X$. For $\varepsilon>0$, we set

$$
V_{\varepsilon}=\{(x, y) \in X \times X: d(x, y)<\varepsilon\} .
$$

Clearly $V_{\varepsilon}[x]=S(x, \varepsilon)$, the sphere with centre $x$ and radius $\varepsilon$. We set $\mathscr{U}_{d}=\left\{U=U^{-1} \subset X \times X: V_{1 / n} \subset U\right.$ for some $\left.n \in N\right\}$. Also for $\mathscr{C} \subset$ $\mathscr{P}(X \times X)$ (the power set of $X \times X)$, we define $\delta=\delta(\mathscr{U})$ by

$$
A \delta B \text { iff }(A \times B) \cap U \neq \phi \text { for each } U \in \mathscr{U} \text {. }
$$

Clearly, if $d$ is a semi-metric on $X$, then $\delta(d)=\delta\left(\mathscr{C}_{d}\right)$.

DEFINITION 1.4. A refining family $\Sigma$ on a toplogical space $(X, \tau)$ is a family $\left\{\alpha_{i}: i \in I\right\}$ of open covers of $X$ such that for each $x \in U \in$ $\tau$, there exists an $i \in I$ such that $\operatorname{St}\left(x, \alpha_{i}\right) \subset U$. In the special case when $I=N, \Sigma$ is called a development on $X$ and the pair $(X, \Sigma)$ is called a developable space. In this case, it is well known that $\Sigma$ may be replaced by another development $\Sigma^{\prime}=\left\{\beta_{j}: j \in N\right\}$ such that if $i<j$ then $\beta_{j} \subset \beta_{i}$; we will assume that $\Sigma$ already satisfies this condition. We will also assume that the developable spaces are $T_{1}$.

LEMMA 1.5. Every refining family $\Sigma$ on a $T_{1}$-space $(X, \tau)$ induces a compatible LO-proximity $\delta=\delta(\Sigma)$ on $X$, where $\delta$ is defined by

$A \delta B \quad$ iff $\operatorname{St}\left(A, \alpha_{i}\right) \cap B \neq \phi$ for each $i \in I$.

Proof. That $\delta$ satisfies 1.1 (i)-(iv), is separated, and is compatible with $\tau$ is obvious. We now show that $\delta$ satisfies 1.1 (vi). Suppose $A \delta B$ and $b \delta C$ for each $b \in B$. Then $A \delta B$ implies that for each $i \in I$, there is a $b \in B$ such that $b \in \operatorname{St}\left(A, \alpha_{i}\right)$. Since St $\left(A, \alpha_{i}\right)$ is open, there is a $j \in I$ such that $b \in \mathrm{St}\left(b, \alpha_{j}\right) \subset \mathrm{St}\left(A, \alpha_{i}\right)$. Since $b \delta C, C \cap \operatorname{St}\left(b, \alpha_{j}\right) \neq$ $\phi$ and this, in turn, implies $C \cap \operatorname{St}\left(A, \alpha_{i}\right) \neq \phi$, i.e., $A \delta C$.

For a developable space $(X, \Sigma), \Sigma=\left\{\lambda_{n}: n \in N\right\}$, we define $d=d(\Sigma)$ by

$$
d(x, y)=\operatorname{Inf}\left\{\frac{1}{n+1}: y \in \operatorname{St}\left(x, \lambda_{n}\right)\right\}
$$

It is easily seen that $d$ is a compatible semi-metric on $X$ and that 
$\delta(d)=\delta(\Sigma)$. For each $n \in N$, we set

$$
B_{n}=\cup\left\{G \times G: G \in \lambda_{n}\right\} .
$$

LEMMA 1.6. $B_{n}=V_{1 / n}$.

Proof. $(x, y) \in V_{1 / n}$ iff $d(x, y) \leqq 1 /(n+1)<1 / n$ iff $y \in \operatorname{St}\left(x, \lambda_{n}\right)$ iff $(x, y) \in B_{n}$.

Definition 1.7. An M-uniformity base $\mathscr{B}$ on $X$ is a family of subsets of $X \times X$ such that,

(i) $\cap\{U: U \in \mathscr{B}\}=\Delta$,

(ii) $U=U^{-1}$ for each $U \in \mathscr{B}$,

(iii) for each $A \subset X$ and $U, V$ in $\mathscr{B}$, there exists a $W \in \mathscr{B}$ such that $W[A] \subset U[A] \cap V[A]$,

(iv) for each $U \in \mathscr{Q}, U \subset V=V^{-1} \subset X \times X$ implies $V \in \mathscr{B}$,

(v) for $A, B$ in $P(X)$ and $U \in \mathscr{B}$, if $V[A] \cap B \neq \phi$ for each $V \in \mathscr{B}$; then there exists an $x \in B$ and a $W \in \mathscr{B}$ such that $W[x] \subset$ $U[A]$. $\quad \mathscr{U} \subset P(X \times X)$ is said to be an $M$-uniformity on $X$ iff there exists a family $\mathscr{B} \subset \mathscr{Q}$ satisfying conditions (i)-(v) above and for each $U \in \mathscr{Q}$ there is a $B \in \mathscr{B}$ such that $B \subset U$.

REMARK 1.8. Mozzochi [12] has shown that an $M$-uniformity $\mathscr{U}$ need not have an open base and that if it does have one, then every convergent filter on $X$ is $\mathscr{C}$-Cauchy.

Definition 1.9. An S-uniformity $\mathscr{Q}$ on $X$ is a family of subsets of $X \times X$ which has a base $\mathscr{B}$ satisfying 1.7 (i)-(iv) and for each $p \in X, B \subset X$ and $U \in \mathscr{B}$, if $V[p] \cap B \neq \phi$ for each $V \in \mathscr{B}$, then there exists an $x \in B$ and a $W \in \mathscr{B}$ such that $W[x] \subset U[p]$.

If $\mathscr{U}$ is an $M$ - or an $S$-uniformity, then $\tau(\mathscr{C} 6)$ is defined as usual; viz. $G \in \tau(\mathscr{Q})$ iff for each $x \in G$, there exists a $U \in \mathscr{C}$ such that $U[x] \subset G$. If $\tau=\tau(\mathscr{C})$, we say that $\tau$ and $\mathscr{U}$ are compatible.

THEOREM 1.10. (See [7], [12].) If थ consists of symmetric subsets of $X \times X$, then $\mathscr{C}$ is an M-uniformity base (resp. S-unformity base) if and only if $\delta(\mathscr{C})$ is a LO-proximity (resp. S-proximity).

Let $(X, d)$ be a semi-metric space and set $V_{1 / n}=\{(x, y): d(x, y)<$ $1 / n\}$. Then $\left\{V_{1 / n}: n \in N\right\}$ is a countable base for a compatible S-uniformity.

2. Semi-metric spaces. In this section we suppose that $(X, d)$ is a semi-metric space and consider the effects of various forms of continuity properties of $d$ on the topology of $X$ and on the proximity $\delta(d)$. 
LEMMA 2.1. In the following (i) and (ii) are equivalent and each implies (iii):

(i) $d$ is separately upper semi-continuous.

(ii) For each $\varepsilon>0$ and $x \in X, S(x, \varepsilon)$ is open.

(iii) $\delta(d)$ is a LO-proximity on $X$.

Proof. (i) $\Rightarrow$ (ii). Let $y \in S(x, \varepsilon)$, i.e., $d(x, y)<\varepsilon$. Since $d$ is upper semi-continuous in $y$, for every $\eta>0$ there is a nbhd. $N_{y}$ of $y$ such that $d(x, z)<d(x, y)+\eta$ for each $z \in N_{y}$. Choose $\eta<\varepsilon-d(x, y)$. Then clearly $N_{y} \subset S(x, \varepsilon)$, showing thereby that $S(x, \varepsilon)$ is open.

(ii) $\Rightarrow$ (i). Suppose $d(x, y)=r$ and $\varepsilon>0$. Clearly $y \in S(x, r+\varepsilon)$, which is open, and hence there exists a nbhd. $N_{y}$ of $y$ such that $N_{y} \subset S(x, r+\varepsilon)$. But this means that for each $z \in N_{y^{\prime}}, d(x, z)<d(x, y)+\varepsilon$, i.e., $d$ is separately upper semi-continuous.

(i) $\Rightarrow$ (iii). Let $\delta=\delta(d)$ and suppose $A \delta B$ and $b \delta C$ for each $b \in B$. Then for each $\varepsilon>0$, there exists an $a \in A$ and a $b \in B$ such that $d(a, b)<\varepsilon$. Since $d$ is upper semi-continuous at $b$, there exists a nbhd. $N_{b}$ of $b$ such that $d(a, x)<\varepsilon$ for each $x \in N_{b}$. Also $b \delta C$ implies the existence of a point $c \in C \cap N_{b}$ and hence, $d(a, c)<\varepsilon$, i.e., $A \delta C$.

COROLlaRY 2.2. If a semi-metric $d$ is separately upper semi-continuous, then $\mathscr{L}_{d}$ is an M-uniformity (obviously with a countable base).

LEMma 2.3. If $d$ is separately lower semi-continuous, then $X$ is regular.

Proof. Let $A$ be closed in $X$ and $p \in X-A$. Then $d(p, A)=$ $r>0$ and hence for each $a \in A, d(p, a) \geqq r$. Since $d$ is lower semicontinuous at $a$, there exists a nbhd. $N_{a}$ of $a$ such that for each $x \in N_{a}, d(p, x)>r / 2$. Set $N_{A}=\cup\left\{N_{a}: a \in A\right\}$. Then $N_{A}$ is a nbhd. of $A$ and $N_{A} \cap S(p, r / 2)=\phi$, thereby showing that $X$ is regular.

The following result is an improvement of a similar one stated by Cook [6]; whereas Cook require the semi-metric $d$ to be continuous we want $d$ to be only separately continuous.

THEOREM $^{1}$ 2.4. If $d$ is separately continuous, then $X$ is Tychonoff.

Proof. Suppose $A$ is a closed subset of $X$ and $x \in X-A$. We may assume that $d(x, A)=1$. As in the proof of Urysohn's lemma, we now show how to construct, for each positive rational $r \in[0,1]$, an open set $V_{r}$ such that $x \in V_{r}$ for each such $r, V_{r}^{-} \subset V_{s}$ whenever $r<s$ and each $V_{r} \subset X-A$. We set $V_{r}=\{y \in X: d(x, y)<r\}$ which is open

1 This result was jointly obtained by Dr. C. M. Pareek and the second author. 
by 2.1 (ii). Also the separate lower semi-continuity of $d$ is equivalent to the set $\{y \in X: d(x, y) \leqq r\}$, being closed for each $r$.

The following theorem is an analogue of the result: A $T_{1}$-space is uniformizable with a countable base iff it is metrizable (and hence has a metric $d$ such that $\delta(d)$ is an $E F$-proximity.)

THEOREM 2.5. A $T_{1}$-space is $M$-uniformizable with a countable base if and only if it has a compatible semi-metric $d$ such that $\delta(d)$ is a LO-proximity.

Proof. Sufficiency is evident from 1.10 and necessity follows from 1.10 and Theorem 4.1.7 of Pareek [15], making use of the remarks just preceding 1.4 .

The followilng analogue of the above result is proved similarly.

THEOREM 2.6. A $T_{1}$-space is S-uniformizable with a countable base if and only if it has a compatible semi-metric $d$ (and obviously $\delta(d)$ is an S-proximity).

3. Developable spaces. In this section we suppose that $(X, \Sigma)$ is a developable space with $\Sigma=\left\{\lambda_{n}: n \in N\right\}$ where each $\lambda_{n}$ is an open cover of $X$ and $\lambda_{n+1} \subset \lambda_{n}$. Let $d=d(\Sigma)$ be the induced semi-metric on $X$. Then $\delta(d)=\delta(\Sigma)$ is a $L O$-proximity on $X$.

LEMMA 3.1. $d$ is upper semi-continuous.

Proof. If $p, q \in X$, we have to consider two cases: (i) $d(p, q)=0$ and (ii) $d(p, q)=1 /(m+1)$ for some $m \in N$. Let $\varepsilon>0$ be arbitrary. In case (i), $\delta(d)$ is a $L O$-proximity by 2.1 and $p=q$. Now choose $n \in N$ such that $1 / n<\varepsilon$; then $p, q \in G$ for some $G \in \lambda_{n}$. For all $(x, y) \in$ $G \times G, d(x, y) \leqq 1 /(n+1)<\varepsilon$. In case (ii), $p, q \in G$ for some $G \in \lambda_{m}$. Then for all $(x, y) \in G \times G, d(x, y) \leqq 1 /(m+1)<1 /(m+1)+\varepsilon$. Thus the result is proved.

The above result (in conjunction with 2.1) provides an alternate proof of the fact that $\delta(\Sigma)$ is a compatible $L O$-proximity and also shows that $\mathscr{U}_{d}$ is a compatible $M$-uniformity with a countable open base $\left\{B_{n}: n \in N\right\}$. This provides a motivation for our next result.

THEOREM 3.2. A $T_{1}$-space is developable if and only if it is $M$ uniformizable with a countable open base.

Proof. Necessity follows from the remarks preceding 3.2 and sufficiency follows from Brown's result ([5], p. 65) that a space is developable iff it has a compatible semi-metric $d$ for which every con- 
vergent sequence is Cauchy, using 1.8.

In [6] Cook states that if a compatible semi-metric $d$ on $X$ is continuous, then $X$ is developable. The following characterization of developable spaces is an improvement of this result.

THEOREM 3.3. $A T_{1}$-space $X$ is developable if and only if it has a compatible upper semi-continuous semi-metric.

Proof. Since the necessity has been proved in (3.1), we need prove only sufficiency. Assuming $d$ to be an upper semi-continuous semi-metric, we prove that $V_{1 / n}$ is open for each $n \in N$ and then the result will follow from (3.2). If $(p, q) \in V_{1 / n}$, then $d(p, q)<1 / n$. Choose $\varepsilon>0$ such that $\varepsilon<1 / n-d(p, q)$. Then from the upper semicontinuity of $d$, there exist nbhds. $N_{p}, N_{q}$ of $p, q$ respectively, such that $d(x, y)<d(p, q)+\varepsilon<1 / n$ for each $(x, y) \in N_{p} \times N_{q}$. This shows that $\left(N_{p} \times N_{q}\right) \subset V_{1 / n}$, showing thereby that $V_{1 / n}$ is open.

Definition 3.4. A proximity base $\mathscr{B}$ for a proximity space $(X, \delta)$ is a family of subsets of $X$ such that if $A \Phi B$, then there exist $C, D \in \mathscr{B}$ such that $A \subset C, B \subset D$ and $C \varnothing D$.

In a $L O$-space, we may assume that the members of $\mathscr{B}$ are closed. The following is an improvement of 2.23 of [12].

Lemma 3.5. If $\mathscr{B}$ is a proximity base for LO-space $(X, \delta)$, then $\left\{U_{A, B}: A, B \in \mathscr{B}\right.$ and $\left.A \phi B\right\}$, where $U_{A, B}=X \times X-[(A \times B) \cup(B \times A)]$, is a base for an $M$-uniformity $\mathscr{U}_{1}$ on $X$.

A developable space $(X, \Sigma)$ is said to be totally bounded iff for each $n \in N$, there exists a finite set $F \subset X$ such that $\operatorname{St}\left(F, \lambda_{n}\right)=X$. It is known that an $E F$-space $(X, \delta)$ has a countable base iff $X$ has a compatible totally bounded metric (Theorem (8.19) of [13]). The following is a partial generalization.

THEOREM 3.6. If a LO-space $(X, \delta)$ has a countable closed base $\mathscr{B}$, then $X$ is a totally bounded developable space.

Proof. $\left\{U_{A, B}: A, B \in \mathscr{B}, A \Phi B\right\}$ is a countable open base for a compatible $M$-unifomity, which is also totally bounded. The result then follows from (3.2).

4. Metrizable spaces. Arkhangel'skii [4] proved that a semimetric space $(X, d)$ is metrizable if $\delta(d)$ is an $E F^{\prime}$-proximity. A glance at his proof shows that the following improved version is true. 
THEOREM 4.1. A $T_{1}$-space is uniformizable with a countable base if and only if it has a compatible semi-metric $d$ such that $\delta(d)$ is an $R$-proximity if and only if it is metrizable.

Nedev [14] proved that a semi-metric space $(X, d)$ is metrizable if $d(A, x)$ is a continuous function of $x$ for each $A \subset X$. The following is an improvement.

THEOREM 4.2. A semi-metric space $(X, d)$ is metrizable if for all closed subsets $A$ of $X, d(A, x)$ is lower semi-continuous.

Proof. Suppose $A$ is closed, $B$ is compact and $A \cap B=\phi$. Since the function $d(A, x)$ is lower semi-continuous and $B$ is compact, it follows that $d(A, B)=d(A, x)$ for some $x \in B$. This implies that $X$ is metrizable (see [4]).

We now give a table which shows the relationship of our results with the classical Alexandroff-Urysohn uniform metrization theorem. Suppose $\left\{W_{n}: n \in N\right\}$ is a countable family of symmetric subsets of $X \times X$ satisfying (a) $\bigcap_{n=1}^{\infty} W_{n}=\Delta$ and (b) for each $x \in X,\left\{W_{n}[x]: n \in N\right\}$ forms a nbhd. base at $x$. Let $d$ be the semi-metric by $\left\{W_{n}\right\}$, namely

$$
d(x, y)=\operatorname{Inf}\left\{\frac{1}{n+1}: y \in W_{n}[x]\right\} .
$$

(We assume without any loss of generality that $W_{n+1} \subset W_{n}$.) Finally set $\mathscr{U}=\left\{U \subset X \times X: W_{n} \subset U\right.$ for some $\left.n \in N\right\}$.

\begin{tabular}{|l|l|l|l|}
\hline $\begin{array}{l}\mathscr{U} \text { is an } \\
\text {-uniformity }\end{array}$ & $\begin{array}{l}\mathscr{U} \text { is an } \\
M \text {-uniformity }\end{array}$ & $\begin{array}{l}\mathscr{U} \text { is an } M \text {-uni- } \\
\text { formity. Each } \\
W_{n} \text { is open }\end{array}$ & $\begin{array}{l}\mathscr{C} \text { is a } \\
\text { uniformity }\end{array}$ \\
\hline $\begin{array}{l}\delta(d) \text { is an } \\
S \text {-proximity }\end{array}$ & $\begin{array}{l}\delta(d) \text { is a } \\
L O \text {-proximity }\end{array}$ & $\begin{array}{l}d \text { is USC } \\
\delta(d) \text { is a } L O \text {-pro- } \\
\text { ximity }\end{array}$ & $\begin{array}{l}\delta(d) \text { is an } \\
E F \text {-proximity }\end{array}$ \\
\hline $\begin{array}{l}X \text { is } \\
\text { semi-metrizable }\end{array}$ & $\begin{array}{l}X \text { is } \\
\text { semi-metrizable }\end{array}$ & $X$ is developable & $\begin{array}{l}X \text { is } \\
\text { metrizable }\end{array}$ \\
\hline
\end{tabular}

5. Metric spaces, developable spaces, semi-metric spaces and mappings connected with them. In this section $f: X \rightarrow Y$ will denote a function from a proximity space $(X, \delta)$ onto a $T_{1}$-space $Y$. When $X$ is developable, semi-metrizable or metrizable, $\delta$ will denote the corresponding naturally induced proximity relation as defined in $\S 1$. Several kinds of functions, which have been defined for the case in which 
$X$ is metrizable, can be redefined more generally when $X$ is a proximity space; this is done by replacing the condition $d(A, B)>0$ by $A \phi B$. These mappings have been systematically discussed in the metric case by Arkhangel'skii [4]. Although we consider their generalizations, for the sake of simplicity we will keep the same terminology and attach $\delta$-before each term ${ }^{2}$.

Our strategy consists in defining a binary relation $\delta^{1}$ on the power set of $Y$ as follows.

$$
E \Phi^{1} F \quad \text { iff } \quad f^{-1}(E) \phi f^{-1}(F)
$$

It is easily verified that $\delta^{1}$ satisfies 1.1 (i)-(iv) and so $\delta^{1}$ is almost a quotient proximity. In order that $\delta^{1}$ be a proximity, naturally we will have to put some additional conditions on $f$; also it is clear that if $\delta^{1}$ is to satisfy stronger proximity conditions, so must $\delta$.

Our first task is to find conditions on $f$ which will make $\delta^{1}$ an $S$-proximity compatible with the topology of $Y$.

Definition 5.2. The function $f$ is called $\delta$-pseudo-open iff for each $y \in Y$ and $A \subset X$, if $f^{-1}(y) \phi(X-A)$ then $y \in \operatorname{Int} f(A)$.

It is easily verified that if $f$ is open or closed, then it is also $\delta$-pseudo-open.

LEMMA 5.3. If $\delta$ is an S-proximity on $X$ and $f$ is $\delta$-pseudo-open, then $y \phi^{1} E$ implies $y \notin E^{-}$.

Proof. $y \phi^{1} E$ implies $f^{-1}(y) \phi f^{-1}(E)$, and since $f$ is $\delta$-pseudo-open, it follows that $y \in \operatorname{Inf} f\left[X-f^{-1}(E)\right]$. But $E \cap f\left[X-f^{-1}(E)\right]=\phi$ and hence $y \notin E^{-}$.

COROLLARY 5.4. If $\delta$ is an S-proximity and $f$ is either open or closed, then $y \Phi^{1} E$ implies $y \notin E^{-}$.

Definition 5.5. The function $f$ is called $\delta$-uniform iff for each $y \in Y$ and each nhbd. $N_{y}$ of $Y, f^{-1}(y) \phi\left(X-f^{-1}\left(N_{y}\right)\right)$.

LEMMA 5.6. If $\delta$ is an S-proximity, then $f$ is $\delta$-uniform if and only if $y \notin E^{-}$implies $y \$^{1} E$.

Proof. If $f$ is $\delta$-uniform and $y \notin E^{-}$, then $f^{-1}(y) \phi f^{-1}(E)$ and by 5.1, $y \phi^{1} E$. Conversely, if $N_{y}$ is a nbhd. of $y$, then $y \notin\left(Y-N_{y}\right)^{-}$ implies $y \phi^{1}\left(Y-N_{y}\right)$ and this is equivalent to $f^{-1}(y) \phi\left(X-f^{-1}\left(N_{y}\right)\right)$. Thus $f$ is $\delta$-uniform.

2 This terminology was suggested by Dr. C. M. Pareek. 
LEMMA 5.7. If $\delta$ is an $R$-proximity and $f$ is continuous and compact, then $y \notin E^{-}$implies $y \phi^{1} E$.

Proof. If $y \notin E^{-}$then $f^{-1}(y) \cap f^{-1}\left(E^{-}\right)=\phi$. Since $f$ is compact, $f^{-1}(y)$ is compact and since $f$ is continuous, $f^{-1}\left(E^{-}\right)$is closed. Finally, $\delta$ being an $R$-proximity on $X, f^{-1}(y) \phi f^{-1}(E)$, which in turn implies $y \phi^{1} E$.

The following result is now obvious.

THEOREM 5.8. The relation $\delta^{1}$ is a compatible S-proximity on $Y$ in the following cases:

(a) $\delta$ is an S-proximity, $f$ is $\delta$-pseudo-open and $\delta$-uniform;

(b) $\delta$ is an $R$-proximity, $f$ is $\delta$-pseudo-open, continuous, and compact.

Next we find out when $\delta^{1}$ is a $L O$-proximity.

THEOREM 5.9. If $\delta$ is a LO-proximity on $X$ and $f$ is $\delta$-uniform and open, then $\delta^{1}$ is a compatible LO-proximity on $Y$.

Proof. By $5.8(\mathrm{~b}), \delta^{1}$ is a compatible $S$-proximity and since $f$ is $\delta$-uniform, it follows that $f$ is continuous. This together with the openness of $f$ implies that for each $B \subset Y, f^{-1}\left(B^{-}\right)=f^{-1}(B)^{-}$. Now $A \delta^{1} B$ iff $f^{-1}(A) \delta f^{-1}(B)$ iff $f^{-1}(A) \delta f^{-1}\left(B^{-}\right)$iff $f^{-1}\left(A^{-}\right) \delta f^{-1}\left(B^{-}\right)$iff $A^{-} \delta^{1} B^{-}$, thereby showing that $\delta^{1}$ is a $L O$-proximity.

To investigate as to when $\delta^{1}$ is an $R$-proximity we introduce a stronger type of map.

Definition 5.10. The function $f$ is called $\delta$-completely uniform iff for each nbhd. $N_{y}$ of $y$ in $Y$, there exists a nbhd. $N_{y}^{\prime}$ of $y$ such that $f^{-1}\left(N_{y}^{\prime}\right) \phi\left(X-f^{-1}\left(N_{y}\right)\right)$.

Since the identity map on $X$ is not $\delta$-completely uniform unless $\delta$ is an $R$-proximity, it is clear that in order to have a meaningful discussion, we must have $\delta$ an $R$-proximity.

THEOREM 5.11. If $\delta$ is an R-proximity on $X$ and $f$ is open and $\delta$-uniform, then $f$ is $\delta$-completely uniform if and only if $\delta^{1}$ is a compatible $R$-proximity.

Proof. To prove the necessity, we first note that Theorem 5.9 shows that $\delta^{1}$ is a compatible $L O$-proximity on $Y$. To see that $\delta^{1}$ is an $R$-proximity, we note that $y \phi^{1} E$ implies $y \in Y-E^{-}$which is open. Since $f$ is $\delta$-completely uniform, there is a nbhd. $N_{y}$ of $y$ such that $f^{-1}\left(N_{y}\right) \phi f^{-1}(E)$ i.e. $N_{y} \phi^{1} E$. Since trivially $y \Phi\left(Y-N_{y}\right)$, it follows that $\delta^{1}$ is an $R$-proximity. 
Conversely, suppose $f$ is open $\delta$-uniform and $\delta^{1}$ is an $R$-proximity. If $N_{y}$ is a nbhd. of $y \in Y, y \phi\left(Y-N_{y}\right)$ and, since $\delta^{1}$ is an $R$-proximity, there exist a set $A \subset Y$ such that $y \phi^{1}(Y-A)$ and $A \phi^{1}\left(Y-N_{y}\right)$, i.e., $y \in \operatorname{Int}(A)$ and $f^{-1}(A) \oiint f^{-1}\left(Y-N_{y}\right)=X-f^{-1}\left(N_{y}\right)$. This proves that $f$ is $\delta$-completely uniform.

In view of Theorem 4.1, it is not necessary to investigate as to when $\delta^{1}$ is an $E F$-proximity and we now turn our attention to the case when $(X, d)$ is a semi-metric space. In this case we suppose that $\delta=\delta(d)$.

DEFINITION 5.12. The function $f$ is called a $T_{1}$-map iff for every pair of distinct points $y_{1}, y_{2}$ of $Y, f^{-1}\left(y_{1}\right) \phi f^{-1}\left(y_{2}\right)$; equivalently, $d\left(f^{-1}\left(y_{1}\right), f^{-1}\left(y_{2}\right)\right)>0$.

LEMmA 5.13. If $(X, d)$ is a semi-metric space and $f$ is a $T_{1}$-map, then

$$
\rho\left(y_{1}, y_{2}\right)=d\left(f^{-1}\left(y_{1}\right), f^{-1}\left(y_{2}\right)\right)
$$

is a semi-metric on $Y$ (not necessarily compatible with the topology of $Y)$.

COROLLARY 5.14. If either $d$ is a metric and $f$ is compact or if $d$ is a semi-metric and $f$ is $\delta$-uniform, then $\rho$ is a semi-metric on $Y$.

LEMma 5.15. Under the conditions of Lemma 5.13, for subsets $A, B$ of $Y$,

$$
\rho(A, B)=d\left(f^{-1}(A), f^{-1}(B)\right)
$$

Proof. $\rho(A, B)=\operatorname{Inf}\left\{d\left(f^{-1}(a), f^{-1}(b)\right): a \in A, b \in B\right\}$. Now

$$
d\left(f^{-1}(A), f^{-1}(B)\right) \leqq d\left(f^{-1}(a), f^{-1}(b)\right)
$$

for each $a \in A, b \in B$ and this implies that $\left(f^{-1}(A), f^{-1}(B)\right) \leqq \rho(A, B)$. Conversely, for every $\varepsilon>0$, there exist $x \in f^{-1}(A), y \in f^{-1}(B)$ such that $d(x, y)<d\left(f^{-1}(A), f^{-1}(B)\right)+\varepsilon$, and hence

$$
\rho(A, B) \leqq d(x, y)<d\left(f^{-1}(A), f^{-1}(B)\right)+\varepsilon,
$$

i.e., $\rho(A, B) \leqq d\left(f^{-1}(A), f^{-1}(B)\right)$, thus proving the result.

CoROLlary 5.16. Under the conditions of Lemma 5.15, $A \delta(\rho) B$ iff $f^{-1}(A) \delta f^{-1}(B)$.

LEMMA 5.17. If $d$ is (resp. separately) upper semi-continuous and 
$f$ is open $\delta$-uniform, then $\rho$ is (resp. separately) upper semi-continuous.

Proof. Let $\rho\left(y_{1}, y_{2}\right)=r$ and let $\varepsilon>0$. Then there are $x_{i} \in f^{-1}\left(y_{i}\right), i=$ 1,2 such that $d\left(x_{1}, x_{2}\right)<r+\varepsilon / 2$. Since $d$ is upper semi-continuous, there exist nbhds. $N_{x_{i}}$ of $x_{i}, i=1,2$ such that for all $z_{i} \in N_{x_{i}}, i=$ $1,2, d\left(z_{1}, z_{2}\right)<r+\varepsilon$. But this shows that $\rho\left(p_{1}, p_{2}\right)<r+\varepsilon$ for $p_{i} \in$ $f\left(N_{x_{i}}\right), i=1,2$ and the result follows since $f$ is open. The case of separate upper semi-continuity is similarly handled.

It is well known that if $X$ is metrizable and $f$ is open $\delta$-uniform, then $Y$ is a developable space. We get an improvement and a more "symmetric" result from (3.3) and (5.17); viz.,

THEOREM 5.18. If $X$ is developable and $f$ is open $\delta$-uniform with respect to the proximity $\delta$ induced by the development on $X$, then $Y$ is also developable.

THEOREM 5.19. If $d$ is a metric on $X$ and if $f$ is continuous, open and compact, then $Y$ is developable.

Proof. By setting $\delta=\delta(d), \delta^{1}=\delta(\rho)$, the result follows from the continuity of $d,(5.8),(5.17)$ and (3.3).

Finally, we conclude with a known result but which follows easily from our analysis.

THEOREM 5.20. If $d$ is a metric on $X$ and if $f$ is open and $\delta$ completely uniform, then $Y$ is metrizable.

Proof. Set $\delta=\delta(d)$ and $\delta^{1}=\delta(\rho)$. It follows from (5.11) that $\delta^{1}$ is a compatible $R$-proximity on $Y$. Also from (5.14), $\rho$ is a semimetric on $Y$ and the compatibility of $\delta^{1}$ implies the compatibility of $\rho$. Hence by (4.1), $Y$ is metrizable.

\section{REFERENCES}

1. P. S. Alexandroff, On some results concerning topological spaces and their continuous mappings, Proc. Symp. Prague (1961), 41-45.

2. - Some results in the theory of topological spaces, obtained within the last twenty-five years, Russian Math. Surveys, 15 (1960), 23-83.

3. - Some basic directions in general topology, Russian Math. Surveys, 19 (1964), 1-39.

4. A. V. Arkhangel'skii, Mappings and spaces, Russian Math. Surveys, 21 (1966), 87114.

5. M. Brown, Semi-metric spaces, Summer Inst. Set Theoretic Topology, Madison, Wisconsin (1957), 64-66.

6. H. Cook, Cartesian products and continuous semi-metrics, Topology Conference, Arizona State University (1967), 58-63. 
7. M. Gagrat, Proximities and extensions of continuous functions, Ph. D. Thesis, I.I.T., Kanpur (1970), (unpublished).

8. M. S. Gagrat and S. A. Naimpally, Wallman compactifications and Wallman realcompactifications, J. Australian Math. Soc., (To appear).

9. — Proximity approach to extension problems, Fund. Math., 71 (1971), 63-76. 10. - Proximity approach to extension problems II, Math. Japonicae, 16 (1971), $35-43$.

11. D. Harris, Regular-closed spaces and proximities, Pacific J. Math., 34 (1970), 675685.

12. C. J. Mozzochi, M. S. Gagrat and S. A. Naimpally, Symmetric generalized topological structures, (1970), (Preprint).

13. S. A. Naimpally and B. D. Warrack, Proximity Spaces, Cambridge Tract In Mathematics No. 59, Cambridge University Press (1970).

14. S. I. Nedev, Continuous and semi-continuous o-metrics, Soviet Math. Dokl., 11 (1970), 975-978.

15. C. M. Pareek, A study of some generalizations of paracompact and metrizable spaces, Ph. D. Thesis, University of Alberta, Canada (1971) (unpublished).

Received July 15, 1971 and in revised form May 1, 1972. The authors wish to thank Dr. C. M. Pareek and the referee for valuable comments and suggestions. Revision was supported by N.R.C. (Canada) grant.

Indian Institute of TeChNOLOGY, KanPUR

UNIVERSITY OF COLORADO

AND

SOUThern IlLOINES UNIVERSITY 



\section{PACIFIC JOURNAL OF MATHEMATICS}

\section{EDITORS}

H. SAMELSON

Stanford University

Stanford, California 94305

C. R. Hовву

University of Washington

Seattle, Washington 98105
J. DugundJI

Department of Mathematics University of Southern California

Los Angeles, California 90007

RICHARD ARENS

University of California

Los Angeles, California 90024

\section{ASSOCIATE EDITORS}

E. F. BECKENBACH

B. H. NeumanN

F. WOLF

K. YoSHIDA

\section{SUPPORTING INSTITUTIONS}

UNIVERSITY OF BRITISH COLUMBIA

CALIFORNIA INSTITUTE OF TECHNOLOGY

UNIVERSITY OF CALIFORNIA

MONTANA STATE UNIVERSITY

UNIVERSITY OF NEVADA

NEW MEXICO STATE UNIVERSITY

OREGON STATE UNIVERSITY

UNIVERSITY OF OREGON

OSAKA UNIVERSITY
UNIVERSITY OF SOUTHERN CALIFORNIA

STANFORD UNIVERSITY

UNIVERSITY OF TOKYO

UNIVERSITY OF UTAH

WASHINGTON STATE UNIVERSITY

UNIVERSITY OF WASHINGTON

AMERICAN MATHEMATICAL SOCIETY

NAVAL WEAPONS CENTER

Printed in Japan by International Academic Printing Co., Ltd., Tokyo, Japan 


\section{Pacific Journal of Mathematics}

\section{Vol. 44, No. $1 \quad$ May, 1973}

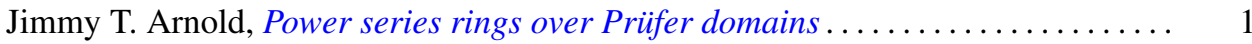

Maynard G. Arsove, On the behavior of Pincherle basis functions . . . . . . . . . 13

Jan William Auer, Fiber integration in smooth bundles ................. 33

George Bachman, Edward Beckenstein and Lawrence Narici, Function algebras

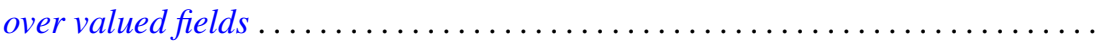

Gerald A. Beer, The index of convexity and the visibility function . . . . . . . . . . .

James Robert Boone, A note on mesocompact and sequentially mesocompact

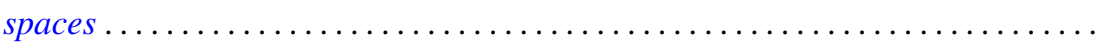

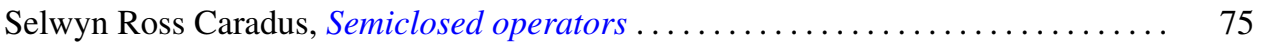

John H. E. Cohn, Two primary factor inequalities . . . . . . . . . . . . . . . 81

Mani Gagrat and Somashekhar Amrith Naimpally, Proximity approach to

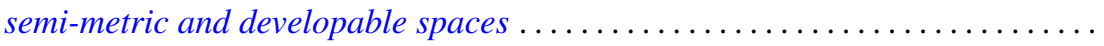

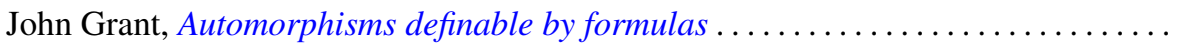

Walter Kurt Hayman, Differential inequalities and local valency ..............

Wolfgang H. Heil, Testing 3-manifolds for projective planes . . . . . . . . . . . . .

107

Melvin Hochster and Louis Jackson Ratliff, Jr., Five theorems on Macaulay

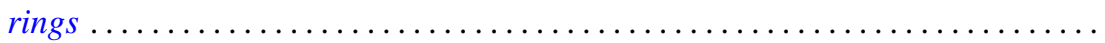

Thomas Benton Hoover, Operator algebras with reducing invariant subspaces ....

James Edgar Keesling, Topological groups whose underlying spaces are separable

Fréchet manifolds...

Frank Leroy Knowles, Idempotents in the boundary of a Lie group . .

191

George Edward Lang, The evaluation map and EHP sequences ...

201

Everette Lee May, Jr, Localizing the spectrum . . . . . . . . . . . .

211

Frank Belsley Miles, Existence of special $K$-sets in certain locally compact abelian groups.

Susan Montgomery, A generalization of a theorem of Jacobson. II . .

T. S. Motzkin and J. L. Walsh, Equilibrium of inverse-distance forces in

three-dimensions.

Arunava Mukherjea and Nicolas A. Tserpes, Invariant measures and the converse

of Haar's theorem on semitopological semigroups .

James Waring Noonan, On close-to-convex functions of order $\beta$

Donald Steven Passman, The Jacobian of a growth transformation

Dean Blackburn Priest, A mean Stieltjes type integral ........ .

Joe Bill Rhodes, Decomposition of semilattices with applications to topological

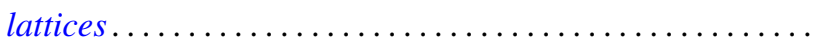

Claus M. Ringel, Socle conditions for $\mathrm{QF}-1$ rings ..........

Richard Rochberg, Linear maps of the disk algebra

Roy W. Ryden, Groups of arithmetic functions under Dirichlet convolution . .

Michael J. Sharpe, A class of operators on excessive functions

Erling Stormer, Automorphisms and equivalence in von Neumann algebras ..

Philip C. Tonne, Matrix representations for linear transformations on series

analytic in the unit disc. 